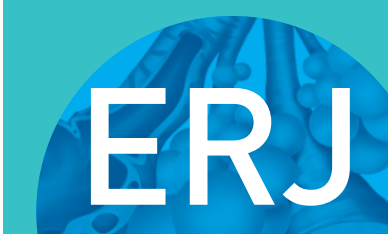

open research

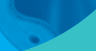

\title{
Physicians' perspectives on communication and decision making in clinical encounters for treatment of latent tuberculosis infection
}

\author{
Claudia C. Dobler (1) $1,2,3,4,5$, Sinthia Bosnic-Anticevich ${ }^{3,6}$ and Carol L. Armour 3,6
}

Affiliations: 'Liverpool Hospital, South Western Sydney Clinical School, University of New South Wales, Sydney, Australia. ${ }^{2}$ Ingham Institute of Applied Medical Research, Sydney, Australia. ${ }^{3}$ Woolcock Institute of Medical Research, University of Sydney, Sydney, Australia. ${ }^{4}$ Centre of Research Excellence in Tuberculosis, University of Sydney, Sydney, Australia. ${ }^{5}$ Mayo Clinic Center for Tuberculosis and Evidence-Based Practice Center, Mayo Clinic, Rochester, MN, USA. ${ }^{6}$ Sydney Local Health District, Sydney, Australia.

Correspondence: Claudia C. Dobler, Dept of Respiratory Medicine, Liverpool Hospital, Elizabeth Street, Liverpool, NSW 2034, Australia. E-mail: c.dobler@unsw.edu.au

ABSTRACT The aim of the study was to explore the views of tuberculosis (TB) physicians on treatment of latent TB infection (LTBI), focusing on decision making and communication in clinical practice.

20 Australian TB physicians participated in a semistructured interview in person or over the telephone. Interviews were recorded, transcribed and analysed thematically.

The study identified challenges that physicians face when discussing treatment for LTBI with patients. These included difficulties explaining the concept of latency (in particular to patients from culturally and linguistically diverse backgrounds) and providing guidance to patients while still framing treatment decisions as a choice. Tailored estimates of the risk of developing TB and the risk of developing an adverse effect from LTBI treatment were considered the most important information for decision making and discussion with patients. Physicians acknowledged that there is a significant amount of unwarranted treatment variation, which they attributed to the lack of evidence about the risk-benefit balance of LTBI treatment in certain scenarios and guidelines that refer to the need for case-by-case decision making in many instances.

In order to successfully implement LTBI treatment at a clinical level, consideration should be given to research on how to best address communication challenges arising in clinical encounters.

@ERSpublications

Overcoming challenges in clinical encounters is essential for implementation of treatment of latent TB infection http://ow.ly/HEFu30izBUu

Cite this article as: Dobler CC, Bosnic-Anticevich S, Armour CL. Physicians' perspectives on communication and decision making in clinical encounters for treatment of latent tuberculosis infection. ERJ Open Res 2018; 4: 00146-2017 [https://doi.org/10.1183/23120541.00146-2017].

Received: Nov 142017 | Accepted after revision: Feb 082018

Conflict of interest: None declared.

Support statement: Claudia C. Dobler was supported by a fellowship from the National Health and Medical Research Council (APP1090198). The project was supported by a research grant from the Ingham Institute of Applied Medical Research and a grant from the Centre of Research Excellence in Tuberculosis Control, University of Sydney. The funders had no role in the study design and analysis. Funding information for this article has been deposited with the Crossref Funder Registry.

The content of this work is @ the authors or their employers. Design and branding are @ERS 2018. This article is open access and distributed under the terms of the Creative Commons Attribution Non-Commercial Licence 4.0. 


\section{Introduction}

Treatment for latent tuberculosis infection (LTBI) is currently promoted as a means to achieve TB elimination in low-incidence settings and contribute to global TB control efforts [1]. Contemporary LTBI guidelines focus on targeted testing and treatment for LTBI in high-risk populations, discourage screening for LTBI in low-risk persons, and refer to case-by-case decision making in persons at intermediate risk of developing TB $[2,3]$. In light of the renewed emphasis placed on LTBI treatment to achieve global TB elimination, it seems timely to take a closer look at current decision making about LTBI treatment at a clinical level, including clinician-patient communication for LTBI treatment in face-to-face encounters.

Discussions with patients about treatment of LTBI are often challenging. Communicating the principle of latency in a way that the patient can understand and convincing an asymptomatic patient of the need for prolonged preventive treatment (over 6-9 months) of a noncontagious infection that may never develop into TB disease, using medications with potential side-effects, is often difficult. Patients' reluctance to accept LTBI treatment and failure to complete a full course of LTBI treatment have been widely recognised as barriers to implementation of this evidence-based intervention [4]. More recently, physicians' impact on unwarranted treatment variation for LTBI, in particular undertreatment, has been increasingly recognised $[5,6]$. The term "unwarranted treatment variation" refers to differences in how patients are treated for the same condition that cannot be explained by different severity of the condition or patient preferences [7]. The Swiss HIV cohort study found that among HIV-infected patients with a positive tuberculin skin test (TST), only $37 \%$ of patients received a full course of LTBI treatment [8]. The study authors speculated that the low LTBI treatment rate was possibly caused by physicians' fear (and by extension patients' fear) of adverse effects and concerns about false-positive TST results [8].

Possible reasons for undertreatment of LTBI include physicians' uncertainty about whether the benefit of treatment outweighs the risks [9] and a perception that LTBI treatment in individuals with LTBI may be beneficial from a public health perspective, but not necessarily from an individual patient's perspective [10]. There is also misinformation, such as the assumption that bacille Calmette-Guérin vaccination offers good protection from developing TB [11]. Physicians can be biased in their judgement, e.g. they assume that LTBI in a migrant from a TB-endemic setting is more likely the consequence of remote infection in the country of origin rather than the result of a known recent close contact with an infectious TB patient [9]. Omission bias, which is the tendency to judge harmful actions as worse or less moral than equally harmful omissions, can be another cognitive bias in this context [12]. Physicians biased in this way could, for example, judge an episode of drug-induced hepatitis caused by LTBI treatment as worse than active TB disease that developed in somebody who did not receive LTBI treatment.

Current guidelines promote treatment of LTBI in high-risk populations (e.g. people with HIV infection), but not in patients at low risk of developing TB [2, 13], and refer to case-by-case decision making about LTBI treatment in populations at intermediate risk of developing TB and lack of evidence about risks and benefits of treatment [2]. The World Health Organization (WHO) guidelines on LTBI acknowledge the paucity of the risk-benefit balance of systematic latent TB testing in several populations, including patients with diabetes, people with harmful alcohol use, tobacco smokers and underweight people, and do not recommend systematic screening and treatment for LTBI in these groups in the absence of additional risk factors [2]. There are some potential discrepancies in recommendations between guidelines, e.g. the WHO guidelines on LTBI do not recommend systematic screening and treatment of LTBI in patients with diabetes [2], whereas the American Thoracic Society/Centers for Disease Control and Prevention recommendations list patients with diabetes as a risk group to be considered for targeted tuberculin testing [3]. Thus, uncertainties remain for physicians who are involved in decision making about treatment for LTBI in individual patients in clinical practice. How clinical decisions about LTBI treatment are made in one-on-one encounters between clinicians and patients may determine whether LTBI treatment can successfully be implemented as a public health measure. This is probably particularly the case in settings with "Western culture" characterised by emphasising a person's right to self-determination and an individualised approach to decision making in medicine. Understanding the drivers of treatment variations from the physicians' perspective is critical to the design of solutions to improve risk-benefit communication for LTBI treatment.

In this study we aimed to evaluate Australian TB physician's perspectives on LTBI treatment, focusing on communication and decision making in the clinical encounter for treatment of LTBI.

\section{Methods}

Ethics

Ethics approval was obtained from the South West Sydney Local Health District Human Research Ethics Committee (HREC/15/LPOOL/114). 


\section{Study setting}

Australia has a low incidence of TB (5.5 per 100000 in 2013) and 88\% of cases occur in the overseas-born population [14]. There is evidence of undertreatment of LTBI, at least in Australia's most populous state of New South Wales, where a retrospective cohort study from 2000 to 2009 showed that $90.5 \%$ of TB contacts (a heterogeneous group of household and nonhousehold contacts) with LTBI did not receive treatment for LTBI, in 95\% of instances because they were not offered treatment for LTBI by the treating physician [15, 16]. Adult migrants from high TB incidence settings who have been identified at pre-migration screening to be at high risk of developing TB are followed up using serial chest radiography, but they are not targeted for LTBI treatment [17]. The majority of TB (and LTBI) care is provided within a network of public chest clinics/TB services in most of Australia's eight states and territories. TB clinics are usually staffed with TB physicians with a medical speciality background in pulmonology or infectious diseases and nurses trained in public health.

There are no national Australian guidelines on LTBI screening and treatment, with the exception of a national policy requiring migrant children aged between 2 and 11 years to complete a TST or interferon- $\gamma$ release assay [18]. Different states may have variable policies and guidelines on LTBI screening and treatment, e.g. in Australia's most populous state of New South Wales there are existing policy directives on performance/indication of TSTs, occupational screening for LTBI among healthcare workers and screening for LTBI in the context of TB contact tracing [19]. The New South Wales policy directive on TB contact tracing states that the recommended management for contacts identified as TST-positive is "either treatment for LTBI or chest $\mathrm{x}$-ray follow up .... In the absence of multi drug resistant (MDR) TB, treatment for LTBI is generally recommended if the person is thought to be recently infected and the risk factors for drug reactions is low" [20].

\section{Study design and participants}

This study took the form of a qualitative study design. We conducted semistructured, in-depth interviews with TB physicians to explore their opinions about LTBI treatment. Eligible participants were Australian physicians who conducted regular TB/LTBI clinics. A purposive snowballing technique was used to identify potential participants. Potential participants were identified either directly based on personal knowledge of one of the study investigators (C.C.D.) or they were identified by chest clinic staff. They were initially contacted by e-mail and received a participant information sheet as well as a consent form for written consent prior to the interview. Participants were recruited from different Australian states and territories, from different medical specialities, and with different levels of experience.

\section{Data collection and analysis}

Interview questions and prompts were developed using an empirical framework based on clinical experience and previous research. Participants' characteristics, including years of experience as a TB physician, medical speciality and state/territory of practice, were gathered prior to the semistructured interviews. Semistructured interviews were conducted by one of the investigators (C.C.D.) in person or over the telephone using an interview guide. Interviews were audiotaped and transcribed verbatim. The interview guide was pilot tested in two participants and some questions were rephrased as a result. We continued interviews until data saturation was reached, i.e. until no new themes emerged that would have enhanced or changed the findings of the study [21]. Data collection ended in June 2016.

Interview content was coded for emerging themes and subthemes using NVivo version 11 qualitative data analysis software (QSR International, Doncaster, Australia). We used the Framework Method with a combined deductive-inductive approach [22]. Some themes and codes (e.g. unwarranted treatment variation) were pre-selected based on the specifics of the research question; other themes and codes emerged during transcript analysis using open coding. Table 1 outlines the categories of unwarranted clinical variations, as defined by John Wennberg, who coined the term [7], and their application to LTBI treatment. Study investigators continuously reflected on the analytic processes and the coding framework was updated to reflect newly revealed themes.

The study results are reported in accordance with the Consolidated Criteria for Reporting Qualitative Research checklist [23].

\section{Results}

26 Australian TB physicians were invited to participate in a semistructured interview; of which 25 agreed to participate and one did not respond to the invitation. After 20 TB physicians were interviewed over the telephone $(n=13)$ or in person $(n=7)$, data saturation was reached and no further interviews were conducted. Participants' characteristics are shown in table 2. The mean \pm SD interview duration was $36 \pm 5.4 \mathrm{~min}$. 
TABLE 1 Unwarranted variations and categories of care [7] applied to treatment of latent tuberculosis infection (LTBI)

\begin{tabular}{|c|c|c|}
\hline $\begin{array}{l}\text { Category of unwarranted } \\
\text { variation applied to LTBI } \\
\text { treatment }\end{array}$ & $\begin{array}{l}\text { Example quotes that indicate } \\
\text { unwarranted treatment } \\
\text { variation }\end{array}$ & Underlying challenge \\
\hline $\begin{array}{l}\text { Effective care } \\
\text { Benefits of LTBI treatment } \\
\text { clearly outweigh the risks, e.g. } \\
\text { close contact of an infectious } \\
\text { TB patient }\end{array}$ & $\begin{array}{l}\text { "I often see the situation where a } \\
\text { TB contact has been found to } \\
\text { have latent TB according to } \\
\text { their Mantoux test, and yet the } \\
\text { decision is made not to offer } \\
\text { treatment because they were } \\
\text { born overseas and have } \\
\text { possibly already been infected } \\
\text { in their home country." }\end{array}$ & $\begin{array}{l}\text { Physicians are prone to cognitive } \\
\text { biases like everybody else }\end{array}$ \\
\hline $\begin{array}{l}\text { Preference-sensitive care } \\
\text { Grey area of risk and benefit, } \\
\text { decision for or against } \\
\text { treatment should be informed } \\
\text { by patient choice, e.g. older } \\
\text { healthcare worker who has } \\
\text { evidence of LTBI }\end{array}$ & $\begin{array}{l}\text { "A difficult patient is a healthcare } \\
\text { worker with a positive skin test. } \\
\text { I think that they're the ones that } \\
\text { are tricky, because I don't think } \\
\text { my management is very } \\
\text { consistent with that type of } \\
\text { patient." }\end{array}$ & $\begin{array}{l}\text { Physicians try to make decisions } \\
\text { for patients in grey areas of } \\
\text { risk and benefit, where ideally } \\
\text { shared decision making should } \\
\text { be used }\end{array}$ \\
\hline $\begin{array}{l}\text { Supply-sensitive care } \\
\text { Frequency of use of the } \\
\text { intervention depends on the } \\
\text { capacity of the local healthcare } \\
\text { system, e.g. LTBI is } \\
\text { undertreated because of lack } \\
\text { of staff in TB clinics }\end{array}$ & $\begin{array}{l}\text { "The immigrants that we } \\
\text { follow-up in the clinic who have } \\
\text { latent TB are probably } \\
\text { undertreated, I think, mainly } \\
\text { because of lack of resources in } \\
\text { terms of nursing, lack of } \\
\text { doctors able to see these } \\
\text { patients and the sheer growth } \\
\text { of the immigrant population." }\end{array}$ & $\begin{array}{l}\text { There is a lack of resources } \\
\text { (infrastructure, human } \\
\text { resources, etc.) to provide } \\
\text { optimal care }\end{array}$ \\
\hline
\end{tabular}

Six themes summarise the main results: "Role of LTBI treatment to achieve TB elimination", "Barriers and facilitators to discussing LTBI treatment with patients", "Physician's role in decision making about LTBI treatment", "Communication style used for LTBI treatment versus other medical conditions", "Communicating risks and benefits of LTBI treatment to patients" and "Warranted and unwarranted variation in LTBI treatment". Figure 1 gives an overview over all themes and subthemes. Illustrative quotes are presented with a pseudonym and sex of interviewees.

\section{TABLE 2 Characteristics of study participants}

$\begin{array}{lr}\text { Subjects } & 20 \\ \text { Sex } & 10(50) \\ \text { Female } & 10(50) \\ \text { Male } & \\ \text { Experience as tuberculosis physician years } & 4(20) \\ \leq 1 & 3(15) \\ 1-5 & 13(65) \\ >5 & \\ \text { Medical speciality } & 15(75) \\ \text { Pulmonary medicine } & 4(20) \\ \text { Infectious diseases } & 1(5) \\ \text { General practice with public health focus } & \\ \text { Australian state/territory of practice } & 11(55) \\ \text { New South Wales } & 3(15) \\ \text { Northern Territory } & 2(10) \\ \text { Victoria } & 2(10) \\ \text { South Australia } & 2(10) \\ \text { Western Australia } & \end{array}$

Data are presented as $\mathrm{n}$ or $\mathrm{n}(\%)$. 


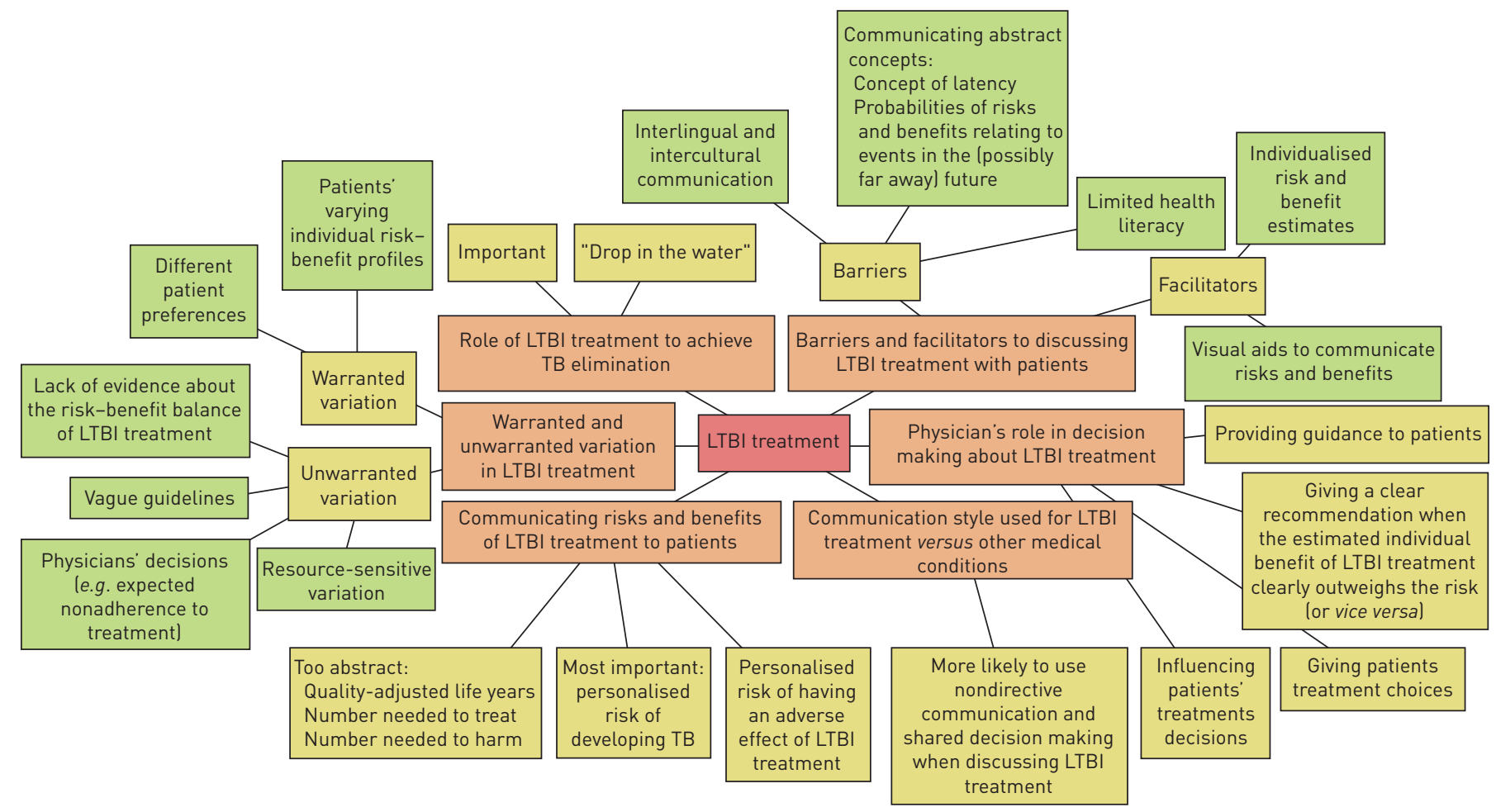

FIGURE 1 Themes and subthemes identified in physician interviews. LTBI: latent tuberculosis infection. Red: central theme; orange: main themes; yellow: subthemes; green: specific topics.

Theme 1: "Role of LTBI treatment to achieve TB elimination"

Most TB physicians thought that treatment of LTBI is an important component of TB control strategies and essential to achieve TB elimination in settings with a low TB incidence, such as Australia. Several TB physicians pointed out the importance of targeting immigrants from high TB incidence settings for LTBI screening and treatment, and emphasised that this population is currently undertreated. There were, however, also critical voices:

Until TB is controlled overseas, we're never gonna achieve elimination... [Physician P, male]

Theme 2: "Barriers and facilitators to discussing LTBI treatment with patients"

Talking to migrants from culturally and linguistically diverse backgrounds, often with the help of interpreters, was identified as a major challenge when discussing the pros and cons of LTBI treatment with patients. In migrants, lack of ability to communicate in English was most commonly considered to be a barrier to effective communication, followed by cultural differences and their impact on health beliefs. Physicians described that communicating abstract concepts of probabilities of risks and benefits relating to events in the (possibly far away) future is often difficult, especially when talking with patients with limited health literacy and/or patients from different cultural backgrounds.

All of us who do this [treatment of LTBI] recognise some of the challenges around communicating some pretty nebulous ideas around risk and latency. And I'm certainly acutely aware of the need to keep developing our capacity to explain these concepts well in a way that people from different cultural backgrounds identify with.

[Physician F, male]

Giving patients risk and benefit estimates that are tailored to their individual epidemiological and medical characteristics was considered to facilitate effective engagement with patients. All physicians agreed that visual aids can help patients understand risks and benefits better. Visual aids that had already been used by $\mathrm{TB}$ physicians included drawings of $\mathrm{TB}$ reactivation risk curves and flow diagrams for the natural progression from latent $\mathrm{TB}$ to $\mathrm{TB}$ disease.

Theme 3: "Physician's role in decision making about LTBI treatment"

Almost all physicians indicated that they would give a clear recommendation for treatment when the estimated individual benefit of the intervention clearly outweighed the risk (or vice versa). 
I'm quite proactive and I guess I try to steer patients towards taking the medication. [Physician H, female]

Many physicians believed that it is part of their professional role to provide guidance on what is likely the best option for an individual patient and not just list the benefits and risks of preventive TB treatment. Physicians were also convinced that patients are looking to them for guidance and expect them to give a recommendation for or against treatment.

They [the patients] come to you for expertise and they want an opinion on what they should do. ... they come with the expectation that you're going to lead them where they should go. [Physician K, male]

People from culturally and linguistically diverse backgrounds as well as patients with low health literacy were thought to expect from physicians that they would make treatment decisions for them rather than to just present choices, which might come across as incompetence.

The majority of physicians, however, also emphasised that they tell patients that they can choose to have or not have LTBI treatment, even when they make a recommendation for one option or the other.

I think even in the most extreme situation it should still be presented as a choice. It's not like a public health undertaking to treat $\mathrm{TB}$ to prevent community harm. [Physician $\mathrm{C}$, male]

The interviewees were aware that they had significant influence on patients' decision for or against treatment and could use this influence if they wanted. As one physician put it:

You can present a case that's very strong, that most people will go along with, in either direction ... [Physician E, male]

Theme 4: "Communication style used for LTBI treatment versus other medical conditions" Physicians stated that they were more likely to use nondirective communication and shared decision making when discussing LTBI treatment with patients compared with discussing the treatment of symptomatic/active diseases (TB as well as noncommunicable diseases). The most commonly mentioned reasons for the use of nondirective communication around LTBI treatment was uncertainty whether a patient would actually ever develop TB disease (and would thus benefit from LTBI treatment) and the perception that, even if patients would develop TB, there would likely be no severe long-term consequences.

I haven't seen anybody have catastrophic problems from not having LTBI treatment. So therefore, when somebody decides not to have treatment, I feel comfortable that that's not a bad decision for them .... Somebody who has poorly controlled asthma [and is not treated], I can see almost an inevitability that they're gonna run into problems, whereas with LTBI they may not. [Physician M, female]

When comparing treatment of LTBI with preventive treatments to reduce cardiovascular risk such as antihypertensives or statins, physicians thought that there was much wider acceptance of preventive treatment for cardiovascular risk among patients as well as the society in general.

\section{Theme 5: "Communicating risks and benefits of LTBI treatment to patients"}

The personalised estimated risk of developing TB was considered the most important information to make a decision about LTBI treatment and to communicate to patients, followed by the estimated risk of developing a significant adverse event, in particular hepatitis, as a consequence of LTBI treatment.

While some physicians would welcome a decision support tool that includes a recommendation for or against LTBI treatment tailored to individual patients' demographic and medical characteristics, the majority of physicians would prefer a decision aid that provides plain estimates of risks and benefits of LTBI treatment, which they could incorporate into the decision-making process and discussion with patients about treatment for LTBI. Communicating a treatment recommendation to patients based on quality-adjusted life years to be gained or lost (obtained by a decision support tool) was considered to be too abstract and thus not helpful by most physicians. The number needed to treat to avoid one case of TB or one TB death and the number needed to harm for one case to develop drug-induced hepatitis or to die from hepatitis were also considered to be too abstract to be communicated to patients by most physicians.

\section{Theme 6: "Warranted and unwarranted variation in LTBI treatment"}

Physicians outlined that a lot of variation in which patients receive LTBI treatment can be explained by patients' varying individual risk-benefit profile as well as by different patient preferences. They did, however, acknowledge that there is a significant amount of unwarranted variation across different Australian states/territories, different clinics and between physicians in the same clinic (see also table 1). 
Day-to-day variation in treatment recommendation for patients with similar demographic and medical profiles by the same physician was also mentioned.

There is a bit of a difference as to how likely you are to have preventive therapy in our clinic depending on which doctor you see. [Physician Q, male]

Lack of evidence about the risk-benefit balance of LTBI treatment in some groups such as migrants from high TB incidence settings without additional risk factors and vague guidelines (which often refer to the need to make decisions about the indication for LTBI treatment on a case-by-case basis in patients at intermediate risk of developing $\mathrm{TB}$ ) were mentioned as main reasons for unwarranted variations in treatment of LTBI. However, physicians acknowledged that unwarranted variations in LTBI treatment were also occurring in instances where the evidence for LTBI treatment was clear, e.g. when treatment was not offered by the physician because of expected nonadherence to LTBI treatment.

I think some of those disparities reflect areas that we need more evidence for. But we also have some individual practitioner and jurisdictional level differences, even where there is clear evidence to inform practice. [Physician E, male]

Resource-sensitive variation in care, referring to less frequently prescribing treatment for LTBI because of limited staff at chest clinics, was mentioned by several participants.

\section{Discussion}

This study describes Australian TB physician's perspectives on LTBI treatment, focusing on the patient-physician encounter. It identifies challenges that physicians face when making decisions and communicating about LTBI treatment, such as only having vague guidelines (referring to the need for case-by-case clinical decision making) for many clinical scenarios, experiencing difficulties in explaining succinctly the abstract concept of latency and disease activation (particularly in an intercultural and interlingual context), and providing guidance to patients while still framing treatment decisions as a choice based on patients' values and preferences.

The findings shed some light on the causes for unwarranted treatment variations for LTBI, in particular undertreatment in a setting with a low TB incidence. They document a need to overcome obstacles at a clinical level, in one-on-one encounters between patients and clinicians, in order to successfully implement LTBI treatment.

In a recent article that called for renewed action to achieve TB elimination in the USA, BAYER and CASTRO [24] emphasised that the prospect of population-level TB elimination should be prioritised over potential clinical risks of LTBI treatment. It is, however, at the clinical level that treatment of LTBI needs to be implemented and therein lies the crux of the matter. Our study findings clearly show that physicians believe that their first responsibility is to help their patients and they will only treat them for LTBI if they are convinced that their patients stand to benefit from this intervention individually or at least that potential harms will not outweigh potential benefits of treatment. This is in line with the WHO guidelines on management of LTBI which state that "Individual benefit outweighing risk should be the mainstay of latent TB testing and treatment" [2]. Achieving wide-scale implementation of LTBI treatment thus relies on convincing physicians and patients that treatment is not simply done for the greater good of the population, but that patients as individuals will, on average, benefit from treatment in most scenarios. Fortunately, the majority of patients with LTBI are likely to have a net benefit from LTBI treatment based on decision analyses modelling [25]. Translating this evidence successfully into clinical encounters, in order to better equip physicians to discuss the risk-benefit profile of LTBI treatment in different scenarios with their patients, might be a key factor in improving the uptake of LTBI treatment.

Research on clinician-patient communication about LTBI treatment has practically been nonexistent so far. This evidence gap is surprising, especially in light of the emerging literature on risk-benefit communication in other areas of preventive medicine (e.g. vaccinations [26], cancer screening [27] and cardiovascular prevention [28]). There might be a perception of LTBI treatment as a public health intervention administered using a programmatic management approach rather than individualised decision making. This perception, however, overlooks the fact that guidelines for LTBI treatment in low-incidence settings often leave room for interpretation in patients at intermediate risk of developing TB and refer to the need for case-by-case decisions at a clinical level based on individual risk-benefit considerations in such scenarios [2], a challenge emphasised by the interviewees in our study.

A major challenge that was highlighted by the interviewed physicians is the communication about LTBI with migrants from culturally and linguistically diverse backgrounds, often with the help of interpreters. As foreign-born persons account for the majority (e.g. in Australia, the USA [14, 29]) or a significant proportion (e.g. in Europe [30]) of all TB cases in high-income countries, physicians will frequently 
encounter these challenges. Even when language barriers can successfully be overcome, cultural differences in illness narratives, values and preferences are likely to influence the perception of latency and preventive treatment to reduce the risk of TB reactivation [31]. A study conducted among Chinese immigrants in British Columbia, Canada, found that study participants often could not differentiate between LTBI and TB disease, and associated the same stigma with LTBI as they did with infectious TB disease [32]. Strategies to create cohesive illness narratives between patients and physicians from different cultural backgrounds deserve further consideration, especially in the context of migrants' health becoming an increasingly important public health concern and health systems struggling to adapt to the needs of international migrants.

The strength of this study is that it provides valuable insights into real-life decision making and communication about LTBI treatment between physicians and patients, which has been underreported to date. The qualitative study design allowed exploration of potential reasons for unwarranted treatment variations and undertreatment of LTBI. A limitation of our study is that the results are not necessarily generalisable to other settings. We do, however, believe that the study describes some core challenges in decision making and communication about LTBI treatment, which are likely relevant to other settings with a low incidence of TB. As purposive rather than random sampling of study participants was used, selection bias cannot be excluded. It is, for example, possible that some of the included TB physicians were primarily known to the study authors (and were therefore contacted for study participation) because of their above average interest in questions about LTBI treatment and their engagement in this area.

The study did not elucidate the specific reasons for the low treatment rate for LTBI previously described in a cohort of TB contacts in New South Wales $[15,16]$. To evaluate specific reasons for the low treatment rate for LTBI among TB contacts, a different study design, possibly including the discussion of case scenarios, might be better suited. Some of the general conclusions from the current study are, however, likely to be relevant in this context as well, in particular the finding that physicians will only prescribe LTBI treatment if they perceive the benefit will outweigh potential harms in an individual patient. This finding emphasises the need for easily accessible evidence on risk-benefit profiles in different clinical scenarios. It would also be interesting to investigate to which extent offering chest radiography follow-up as an alternative to LTBI treatment in TB contacts [20] and migrants identified at high risk of developing $\mathrm{TB}$ at pre-migration screening [17] might be contributing to low LTBI treatment rates.

The use of a communication and decision tool to facilitate shared decision making between physicians and patients could possibly address some of the challenges that physicians experience when discussing LTBI treatment with patients. Shared decision making, sometimes supported by specific tools (decision aids or communication tools) for the patient-physician encounter, refers to patients and clinicians engaging in a deliberative dialogue, weighing up the pros and cons of different treatment option to arrive at the best possible solution to address the patient's healthcare situation [31]. Shared decision making has been described as a possible solution to translating evidence from the population/public health level to the individual patient in clinical practice [33]. The use of decision aids for shared decision making also has the potential to reduce unwarranted variations [34].

Decision aids for shared decision making during the clinical encounter often visually display an individualised risk-benefit profile tailored to an individual patient, effectively communicating the information considered most important by the interviewed physicians (i.e. the individual's risk of developing TB and of having a side-effect from LTBI treatment). In which way tailored risk communication impacts on uptake and completion of LTBI treatment is, however, unknown. In cancer screening, results are mixed regarding the effect of individualised health messages on patient behaviour (e.g. uptake of screening) [35].

In conclusion, there are many challenges associated with decision making and communication regarding LTBI treatment in clinical practice. Further research is required to fill the evidence gap on how to reduce unwarranted treatment variation in patients with similar risk-benefit profiles and how to best communicate risks and benefits of LTBI treatment to patients from culturally and linguistically diverse backgrounds and patients with limited health literacy. The use of communication tools/decision aids during the patient-physician encounter deserves further consideration.

Acknowledgement: We would like to thank the participants in the study for the time given and for sharing their views and experiences. Without their participation, this research would not have been possible.

\section{References}

1 Lonnroth K, Migliori GB, Abubakar I, et al. Towards tuberculosis elimination: an action framework for low-incidence countries. Eur Respir J 2015; 45: 928-952. 
2 World Health Organization. Guidelines on the Management of Latent Tuberculosis Infection. WHO/HTM/TB/ 2015.01. Geneva, WHO, 2015.

3 American Thoracic Society, Centers for Disease Control and Prevention. Targeted tuberculin testing and treatment of latent tuberculosis infection. Am J Respir Crit Care Med 2000; 161: S221-S247.

4 Horsburgh CR Jr, Goldberg S, Bethel J, et al. Latent TB infection treatment acceptance and completion in the United States and Canada. Chest 2010; 137: 401-409.

5 Dobler CC. Unwarranted prescription variations for treatment of latent tuberculosis infection. Lancet Infect Dis 2017; 17: 134.

6 Alsdurf H, Hill PC, Matteelli A, et al. The cascade of care in diagnosis and treatment of latent tuberculosis infection: a systematic review and meta-analysis. Lancet Infect Dis 2016; 16: 1269-1278.

7 Wennberg JE. Unwarranted variations in healthcare delivery: implications for academic medical centres. BMJ 2002; 325: 961-964.

8 Elzi L, Schlegel M, Weber R, et al. Reducing tuberculosis incidence by tuberculin skin testing, preventive treatment, and antiretroviral therapy in an area of low tuberculosis transmission. Clin Infect Dis 2007; 44: 94-102.

9 Dobler CC, Luu Q, Marks GB. What patient factors predict physicians' decision not to treat latent tuberculosis infection in tuberculosis contacts? PLoS One 2013; 8: e76552.

10 Degeling C, Denholm J, Mason P, et al. Eliminating latent tuberculosis in low-burden settings: are the principal beneficiaries to be disadvantaged groups or the broader population? J Med Ethics 2017; 43: 632-636.

11 Hirsch-Moverman Y, Tsiouris S, Salazar-Schicchi J, et al. Physician attitudes regarding latent tuberculosis infection: international vs. U.S. medical graduates. Int J Tuberc Lung Dis 2006; 10: 1178-1180.

12 Aberegg SK, Haponik EF, Terry PB. Omission bias and decision making in pulmonary and critical care medicine. Chest 2005; 128: 1497-1505.

13 American Thoracic Society. Targeted tuberculin testing and treatment of latent tuberculosis infection. MMWR Recomm Rep 2000; 49: 1-51.

14 Toms C, Stapledon R, Waring J, et al. Tuberculosis notifications in Australia, 2012 and 2013. Commun Dis Intell Q Rep 2015; 39: E217-E235.

15 Dobler CC, Marks GB. Risk of tuberculosis among contacts in a low-incidence setting. Eur Respir J 2013; 41: 1459-1461.

16 Dobler CC. What do we know about the outcomes of tuberculosis contact investigations in NSW? NSW Public Health Bull 2013; 24: 34-37.

17 Chan IHY, Kaushik N, Dobler CC. Post-migration follow-up of migrants identified to be at increased risk of developing tuberculosis at pre-migration screening: a systematic review and meta-analysis. Lancet Infect Dis 2017; 17: 770-779.

18 Department of Home Affairs. Understanding immigration health changes for 20 November 2015. 2015. www. homeaffairs.gov.au/Visasupport/Pages/br3-health-client.aspx Date last accessed: January 8, 2018.

19 NSW Ministry of Health. Policy directives, guidelines and information bulletins. www.health.nsw.gov.au/policies/ Pages/default.aspx Date last accessed: January 8, 2018.

20 NSW Ministry of Health. Tuberculosis contact tracing. policy directive PD 2008_017. 2008. www1.health.nsw.gov. au/pds/ActivePDSDocuments/PD2008_017.pdf Date last accessed: January 8, 2018.

21 Patton MQ. Qualitative Evaluation and Research Methods. Newbury Park, Sage, 1990.

22 Gale NK, Heath G, Cameron E, et al. Using the framework method for the analysis of qualitative data in multi-disciplinary health research. BMC Med Res Method 2013; $13: 117$.

23 Tong A, Sainsbury P, Craig J. Consolidated criteria for reporting qualitative research (COREQ): a 32-item checklist for interviews and focus groups. Int J Qual Health Care 2007; 19: 349-357.

24 Bayer R, Castro KG. Tuberculosis elimination in the United States - the need for renewed action. N Engl J Med 2017; 377: 1109-1111.

25 Dobler CC, Martin A, Marks GB. Benefit of treatment of latent tuberculosis infection in individual patients. Eur Respir J 2015; 46: 1397-1406.

26 Leask J, Kinnersley P, Jackson C, et al. Communicating with parents about vaccination: a framework for health professionals. BMC Pediatr 2012; 12: 154

27 Barrett B, McKenna P. Communicating benefits and risks of screening for prostate, colon, and breast cancer. Fam Med 2011; 43: 248-253.

28 Navar AM, Stone NJ, Martin SS. What to say and how to say it: effective communication for cardiovascular disease prevention. Curr Opin Cardiol 2016; 31: 537-544.

29 Centers for Disease Control and Prevention. Reported Tuberculosis in the United States, 2014. Atlanta, Department of Health and Human Services, 2015.

30 Hollo V, Beaute J, Kodmon C, et al. Tuberculosis notification rate decreases faster in residents of native origin than in residents of foreign origin in the EU/EEA, 2010 to 2015. Euro Surveill 2017; 22: 30486.

31 Dobler CC, Spencer-Bonilla G, Gionfriddo MR, et al. Shared decision making in immigrant patients. Cureus 2017; 9: e1461.

32 Gao J, Berry NS, Taylor D, et al. Knowledge and perceptions of latent tuberculosis infection among Chinese immigrants in a Canadian urban centre. Int J Fam Med 2015; 2015: 546042.

33 Politi MC, Wolin KY, Legare F. Implementing clinical practice guidelines about health promotion and disease prevention through shared decision making. J Gen Intern Med 2013; 28: 838-844.

34 O'Connor AM, Llewellyn-Thomas HA, Flood AB. Modifying unwarranted variations in health care: shared decision making using patient decision aids. Health Aff 2004; Suppl. Variation: Var63-Var72.

35 Trevena LJ, Zikmund-Fisher BJ, Edwards A, et al. Presenting quantitative information about decision outcomes: a risk communication primer for patient decision aid developers. BMC Med Inform Decis Mak 2013; 13 : S7. 